resolved. Wenrich (1916) found that in grasshoppers particular chromomeres could be identified by their size and position on the chromosome. Belling (1931) identified the chromomeres with the genes, and attempts at counting them in various plants have given values ranging roughly from 1000 to 2500 . The chromosome threads become spiral in various stages of mitosis, and Kuwada has found indications of a spiral within the spiral. Others find the spiral chromonema splitting lengthwise in prometaphase.

In what may be called the external morphology of the chromosomes more marked progress has been made. In 1912, S. Navashin discovered that certain chromosomes in Galtonia have a tiny more or less globularsatellite attached to oneend by a thread. One or more pairs of satellited chromosomes have since been observed in many plants and animals.

The spindle fibre attachment constriction is now a well-recognised feature of all plant chromosomes, and 'kinetic bodies', 'knobs', 'heads', additional constrictions, vesicles and their special features have been observed. Levitsky, M. Navashin and others of the Russian school have been active in comparing the karyotypes of various plant groups.

The nature of the nucleolus has long been a mystery, but light has recently been thrown on this problem. Wenrich (1916) noted in the meiotic nuclei of a grasshopper that the nucleolus bore a constant relation to a particular pair of chromosomes. Later a definite 'nucleolar body' was discovered as a darker staining area of the nucleolus in pollen mother cells of Lathyrus (Latter, 1926), and it was shown that a loop of the chromosome thread was constantly attached to it. The same condition has since been found in Lathroea, Oenothera, Malva and rice, and frequently two such bodies rather than one are attached to the nucleolus. S. Navashin had observed a pair of satellites attached to the nucleolus, and in studies of maize, McClintock (1931) has shown that the satellited pair of chromosomes is concerned in producing the nucleolus in teleophase at a particular locus. When these chromosomes are widely separated, two nucleoli will be produced. Heitz (1931) has observed similar conditions in Vicia and other plants as well as in insects (1933), while Dearing (1934) shows in the amphibian Amblystoma the same relation between a satellited pair of chromosomes and the (usually two) nucleoli present. Thus it appears to be the function of a particular locus of one pair of chromosomes to produce the nucleolus, but this is not the whole story. In this, as in other fields of nuclear study, the results in animals and plants have been re. markably synchronised.

Lack of space prevents more than mention of the important method of micro-dissection, by which living cells can be dissected with glass needles under an immersion lens. Introduced by Kite and Chambers in 1912, it has led to many interesting observations of the physical condition of various cell constituents, including the chromosomes.

Finally, we may refer to the investigations of the chromosome structure in the salivary glands of insects. These giant chromosomes have long been known to show a banded structure, but its significance has only been brought out by the recent observations of Painter, Bridges, Koltzoff and others. The discs differ markedly in details of structure, but they are definitely spaced at varying intervals along the chromosomes, and from the evidence of their pairing and genetio behaviour it is clear that they must be identified in some way with the genes. Certain of the bands have been shown to vary according to the genetic make-up of the animal. Bridges has just published $(J$. Hered., 26, No. 2) maps of the four chromosomes (significantly separated into six) in the salivary glands of Drosophila melanogaster, identifying each band and also pointing out many other structural features or landmarks at various loci of the chromosomes. These chromosomes total about $1180 \mu$, or 150 times the length of the meiotic chromosomes. The bands, which probably represent loci, number 2650 , the little fourth chromosome having 34 . This begins a new era in chromosome study.

\title{
Chemistry of the Anthocyanins
}

\section{By Prof. R. Robinson, F.R.s., Waynflete Professor of Chemistry, University of Oxford}

$\mathrm{T}$ Elassical paper of Willstätter and Everest (1913), on the isolation of the pigment of blue cornflowers, heralded a dramatic transformation of the state of our knowledge of the blue and red colouring matters of flowers and blossoms, and the present position is that we not only know the molecular structure of the more important and widespread anthocyanins, but also that many of them have been made artificially in the laboratory. With the simultaneous growth of precise information about chlorophyll, the carotenoids, the polysaccharides and the terpenes, one may say that all the more obvious challenges of vegetative Nature to the organic chemist have been taken up, and taken up successfully. Deep mysteries there are still, it is true, but one must probe 
beneath the surface in order to find them. Willstätter owed his triumph largely to recognition of the fact that the anthocyanins, although nonnitrogenous, form salts with strong acids, and these salts can be purified by means of the technique appropriate to many ammonium salts, that is, solution in a hydroxylic solvent and precipitation with a non-hydroxylic solvent.

An early observation records the unconscious use of formic acid for the extraction of an anthocyanin. J. Wray (1670) wrote: "Bare an anthill with a stick and then cast the [chicory] flowers upon it, and you shall see the ants creep very thick over them. Now as they creep, they let fall a drop of liquor from them, and where that chanceth to light, there you shall have in a moment a large red stain". This was followed up and red extracts of blue chicory flowers were made with acidic solutions.

Willstätter and his colleagues extracted the anthocyanin salts by means of methyl alcoholic hydrogen chloride, acetic acid or other similar solvents, and precipitated a crude, often syrupy, product with ether. The process was usually repeated, perhaps with variation of solvent, and until the anthocyanin could be caused to separate from aqueous or alcoholic solution as a chloride or picrate. Purification was then possible by crystallisation, or a series of separations leading to eventual crystallisation. The different examples offered very divergent degrees of difficulty and indeed, in some cases, the skill of the florist in the breeding of deeply-coloured varieties has been such that the dried petals can almost be regarded as crude anthocyanin.

The blue cornflower and the red poppy represent the more difficult type, whereas the colouring matter is readily isolated from special deep red varieties of dahlias and chrysanthemums and from certain garden violas. The dried petals of the blue-black viola (Viola tricolor) employed by Willstätter and Weil (1916) for the isolation of violanin contained no less than 24 per cent of this pigment.

Willstätter was fortunate in the selection of material because among the first flowers studied were to be found representatives of the three main types in the group. From the cornflower, the rose and the dahlia he obtained cyanin chloride, $\mathrm{C}_{27} \mathrm{H}_{31} \mathrm{O}_{16} \mathrm{Cl}$, which splits up on hydrolysis into cyanidin chloride, $\mathrm{C}_{15} \mathrm{H}_{11} \mathrm{O}_{6} \mathrm{Cl}$, and two molecules of glucose; from the scarlet pelargonium he isolated pelargonin chloride, $\mathrm{C}_{27} \mathrm{H}_{31} \mathrm{O}_{15} \mathrm{Cl}$, which similarly yields on hydrolysis two molecules of glucose and pelargonidin chloride, $\mathrm{C}_{15} \mathrm{H}_{11} \mathrm{O}_{5} \mathrm{Cl}$, whilst the wild purple larkspur afforded delphinin chloride, $\mathrm{C}_{41} \mathrm{H}_{39} \mathrm{O}_{21} \mathrm{Cl}$, which gives, on hydrolysis, delphinidin chloride, $\mathrm{C}_{15} \mathrm{H}_{11} \mathrm{O}_{7} \mathrm{Cl}$, along with two molecules of glucose and two molecules of $p$ hydroxybenzoic acid. [Anthocyanins containing acyl groups, often $p$-coumaric acid (Karrer), are widely distributed and are termed complex antho. cyanins. The true analogue of pelargonin chloride and cyanin chloride in the delphinidin series has recently been isolated from Salvia patens (Reynolds, Scott-Moncrieff and R. R., 1934); it is termed delphin chloride and has the composition $\mathrm{C}_{27} \mathrm{H}_{31} \mathrm{O}_{12} \mathrm{Cl}$.]

The aglucones were termed anthocyanidins, and in addition to the three already mentioned only certain of their methyl ethers have been encountered. The two or three exceptional cases serve merely to establish the rule that all the anthocyanins are derivatives of the three basic types. Further work showed that the aglucones may be combined with one molecule of glucose or of galactose, or with a rhamnoglucose or aldopentoglucose, and that isomeric diglucosides exist. Thus mecocyanin chloride (from Papaver rhoeas) is quite different from cyanin chloride, but like it has the composition $\mathrm{C}_{27} \mathrm{H}_{31} \mathrm{O}_{16} \mathrm{Cl}$, and it also furnishes cyanidin chloride and two molecules of glucose on hydrolysis.

The probable nature of the difference between pelargonidin, cyanidin and delphinidin is clearly indicated by the results of fusion with potash. All three give phloroglucinol (I) as one of the products,

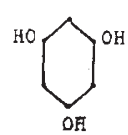

(I)

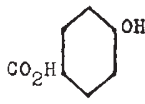

(II)

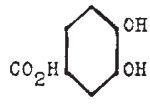

(III)

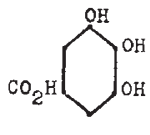

(IV) but pelargonidin was in addition degraded to $p$-hydroxybenzoic acid (II), cyanidin to protocatechuic acid (III) and delphinidin to gallic acid (Iv).

Taking into consideration the results of precedent investigations on natural flavones (luteolin, v) and flavonols (quercetin, vi) with which the names of Kostanecki, A. G. Perkin and Herzig are chiefly associated, and also the accumulated knowledge of the properties of flavylium salts (such as VII) (Collie, Werner, Bülow, Decker, W. H. Perkin and R.R.), it was apparent that the facts pointed to the formula VIII for cyanidin chloride. The formulæ for the other antho. cyanidins would be derived by modifications of the group $\mathrm{A}$ in vIII to conform with the results of potash fusion as mentioned above.

This surmise was quickly justified by the discovery that flavones and flavonols could be reduced by metals in acid solution with the 
formation of oxonium salts having the reactions of anthocyanidins (Everest, Willstätter). In particular, Willstätter and Mallison showed that quercetin gives a small yield $(0.6 \mathrm{gm}$. from $30 \mathrm{gm}$.) of pure cyanidin chloride when it is reduced in<smiles>OC1CCC(C2=CCC3CCCCC3O2)CO1</smiles><smiles>OC1CC2OC3(O)CCOCC3(C3CCC(O)C3)OC2C1</smiles><smiles>OC1CCC2CCC(C3CCCC3)OC2C1</smiles>

(VII)<smiles>OC1CCC(C2CCC3CCCCC3O2)CC1C1CCCCC1</smiles>

(VIII)

aqueous methyl alcoholic hydrochloric acid by means of magnesium (VI $\rightarrow$ VIII). This was followed up by a more formal synthesis of pelargonidin (Willstätter and Zechmeister), whilst the present writer and his collaborators have synthesised all the anthocyanidins, including the methyl ethers, by convenient and generally applicable methods.

The naturally occurring methyl ethers of the anthocyanidins are peonidin chloride (Ix), the aglucone of peonin, a diglucoside, and oxycoccicyanin, a monoglucoside; petunidin chloride (x), the aglucone of petunin chloride, a diglucoside; malvidin chloride (XI), the aglucone of a monoglucoside, a monogalactoside, a diglucoside, and of complex anthocyanins; hirsutidin chloride (xII), found only as the aglucone of hirsutin chloride, a diglucoside of Primula hirsuta (Karrer). Of these, malvidin, also called syringidin and œnidin, is of such frequent occurrence that it almost deserves recognition as a fourth fundamental type. Its monoglucoside is œnin, the pig-<smiles>OC1CCC(C2CCC3CCCCC3O2)C(O)C1</smiles>

(IX)<smiles>OC1CCC(C2CCC3CCCCC3O2)CC1O</smiles>

(XI)

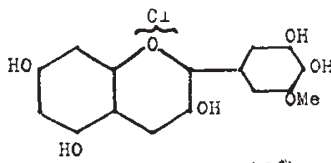

$(x)$

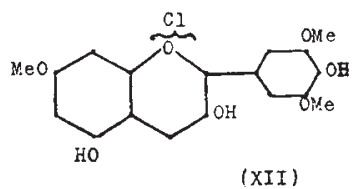

(XII)

ment of black grapes, one of the most frequently studied anthocyanins.

In the earlier work on anthocyanidin syntheses (Pratt, R.R.) it was thought necessary to protect the nuclear hydroxyl groups very completely, and

the products were finally demethylated by means of hot hydriodic acid. Such a process could clearly not be applicable to the methyl ethers (IX-XII), and a technique has been gradually evolved that allows of the minimum of protection, and that by means of acyl groups only. The scheme below illustrates the stages of the synthesis of malvidin chloride by the best-known method.

Phloroglucinaldehyde (XIII) may be synthesised from carbon via five isolated intermediate stages, namely, acetylene, benzene, nitrobenzene, trinitrobenzene, phloroglucinol ; hydrocyanic acid is also used and may be obtained from carbon in two stages. The intermediate $\mathrm{XV}$ may be synthesised from carbon in thirteen isolated stages, namely, acetylene, benzene, benzoic acid (better in one more stage), disulphobenzoic acid, 3 : 5-dihydroxybenzoic acid, bromodihydroxybenzoic acid, gallic acid, trime-
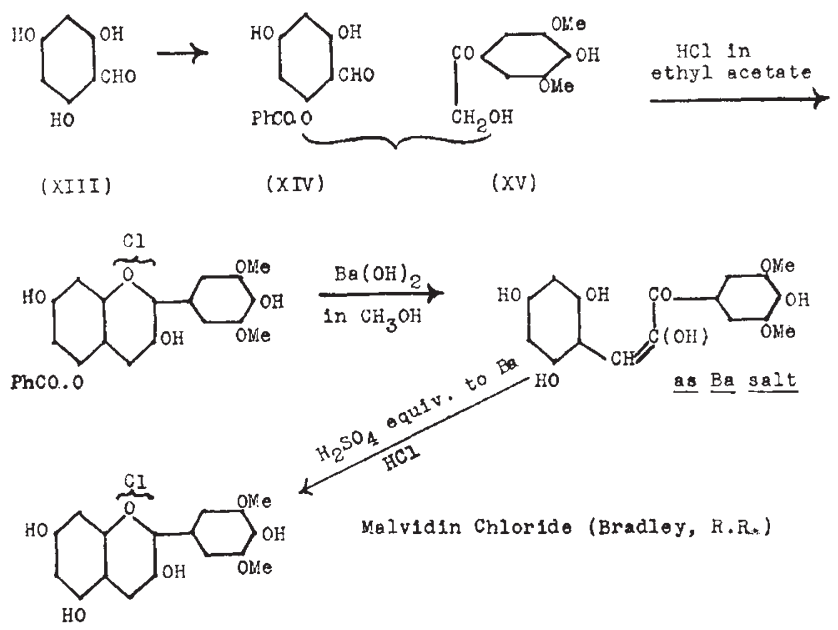

thoxybenzoic acid, syringic acid, acetylsyringic acid, acetylsyringoyl chloride, diazodimethoxyacetoxyacetophenone, $\omega$-4-diacetoxy-3:5-dimethoxy-acetophenone; two carbon atoms are introduced by way of carbon monoxide, methyl alcohol, methyl sulphate, and one from carbon monoxide, methyl alcohol, methylamine, methylurea, nitrosomethylurea, diazomethane.

The monobenzoylphloroglucinaldehyde (xIv) has been found to be a particularly convenient first component in all syntheses of the above type, and its use (Robertson, R. R.) facilitated the synthesis of the anthocyanins themselves. Hints in regard to the site of the sugar residues in the anthocyanins had been obtained from a study of their colour reactions and other properties (Willstätter, Karrer, R. R. and their colleagues) in comparison with synthetic, analogous flavylium salts, but it is unnecessary, in this brief article, to recount the arguments, especially as none of them was conclusive. The case is one in which synthesis has 
been applied not merely to the confirmation but also to the determination of structure.

Nor is it necessary to mention the several stages of approach to the synthetic method, which is identical in principle with that already described. It was a question of applying the art of the organic chemist to the preparation of the various glucosida.ted intermediates of types XIV and XV. For example, by suitable modifications the four possible isomeric $\beta$-glucosides of pelargonidin were

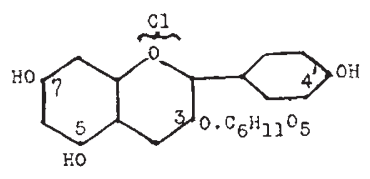

$(X V I)$

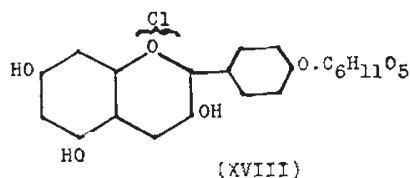

(XVIII)
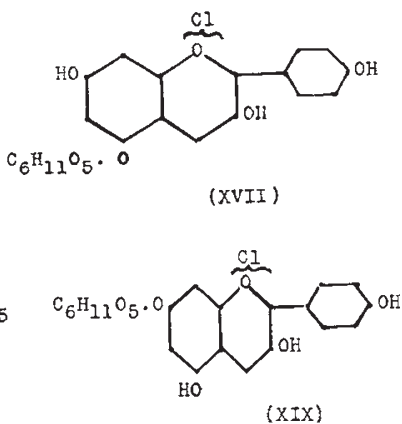

synthesised (xVI, xvII, xVIII, xIx) (Robertson, Léon, Seshadri, R. R.) ; xvI was found to be identical with Willstätter's callistephin and xVII with his pelargonenin, not a natural anthocyanin but the first product of the hydrolysis of the diglucoside, pelargonin.

The natural monoglucosidic anthocyanins are all constituted like xVI. The naturally occurring mono-glycosides synthesised are callistephin (from the aster or red carnation), chrysanthemin (cyanidin 3-glucoside from the chrysanthemum and other flowers), oxycoccicyanin (peonidin 3-glucoside from American cranberries), œnin (malvidin 3-glucoside from purple-black grapes), fragarin (pelargonidin 3-galactoside from strawberries), idæin (cyanidin 3-galactoside from European cranberries), primulin (malvidin 3galactoside from Primula sinensis). The lastmentioned case is of interest because it illustrates the value of one of the methods used for the characterisation of anthocyanins. The monoglucosides are distributed between dilute hydrochloric acid and isoamyl alcohol, but the distribution number varies with the concentration. By plotting the logarithms of the concentrations in the aqueous and alcoholic layers against each other, a straight line is obtained with a slope of 2 ; this indicates that double molecules exist in the water and single molecules in the isoamyl alcohol. The curves obtained in this way are much more reliable than single determinations of the distribution numbers because they afford evidence of homogeneity.

In connexion with work on the bilberry pigments, malvidin 3-galactoside was synthesised (Bell, R. R.), and its distribution properties were found to differ from those of œnin (malvidin 3 -glucoside), so that a study of malvidin 3glycosides containing unidentified sugar residues was feasible. Cyclamin (from cyclamen, Karrer) was found to be identical with œnin, but primulin (from $P$. sinensis), isolated by Miss R. ScottMoncrieff, tallied with the galactoside. Closely related to the monoglycosides are the true biosides, and one example has been cleared up by synthesis. Cyanidin 3-cellobioside, 3-maltoside, 3-lactoside and 3-gentiobioside were synthesised (Inubuse, Grove and R. R.) and the latter was found to be identical with Willstätter's mecocyanin. The rhamnoglucosides have not yet been prepared in the laboratory but their reactions are closely similar to those of known 3-saccharides.

There remain the diglucosidic anthocyanins par excellence-pelargonin, cyanin, malvin, etc. At first regarded as biosides, these are now known to be di-monoglucosides, and the two sugar residues are attached to different hydroxyl groups of the anthocyanidin molecules.

The suggestion that they are $3: 5$-diglucosides was first put forward in a letter to the Editor of Nature (G. M. R. and R. R.) and it was quickly confirmed by synthesis. The glucose rests must be introduced into both components. Normally and preferably these were acetylated, and the product was submitted to hydrolysis by alkali and reconstituted by the action of hydrochloric acid.

It is perhaps a matter of interest that cyanin chloride (xx) has been synthesised directly from unprotected components (Resuggan, R. R.).

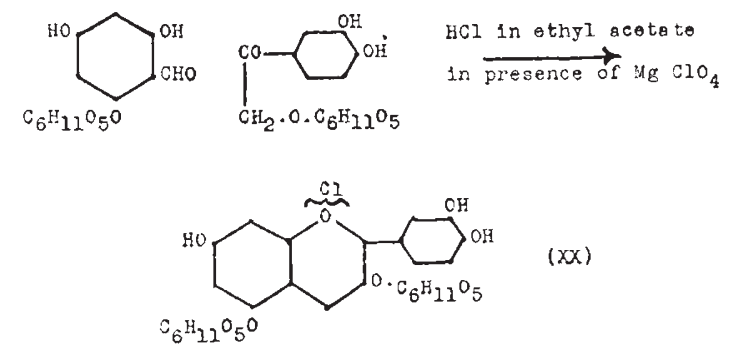

The naturally occurring 3:5-diglucosides synthesised include pelargonin, cyanin, peonin, malvin and hirsutin (Todd and R. R.) ; the additional case of delphin has been mentioned already.

The possession of pure synthetic specimens of the anthocyanins and anthocyanidins has made it possible to devise simple tests for the rapid recognition of the nature of the pigments in crude extracts of plant material, and a survey (G. M. R. and R. R.) has been made of a wide variety of flowers and other parts of plants in respect of their 
anthocyanin content. In general, we are able to specify the anthocyanidin and the position of the sugar groups, but the latter cannot be identified (except for rhamnose and the aldopentoses). This survey has made the dominating position of pelargonidin, cyanidin, delphinidin and malvidin even more clear, but it has also disclosed the occurrence in Nature of certain widely distributed anthocyanins not yet isolated in substance. As examples, the pelargonidin 3-bioside of the orangered nasturtium and the pelargonidin 3-rhamnoglucoside of the scarlet gloxinia may be cited. The orange-scarlet flowers of Gesnera fulgens were found to contain a new anthocyanin termed gesnerin ; it is apigeninidin 5-glucoside (XxI) and has been synthesised (Todd, G. M. R. and R. R.) ; it is the only known anthocyanin related to a flavone rather than to a flavonol, although carajurin (XXII), a crystalline constituent of a cosmetic pigment used by the natives of the Orinoco, is a colour-base of such a substance (Chapman, A. G. Perkin and R. R.) Other special types of anthocyanins are the nitrogenous pigments of Beta, Bougainvilloea, Amaranthus, Celosia, etc.; their nature has not been fully elucidated; also the bright yellow water-soluble colouring matters of Papaver alpinum and $P$. nudicaule.

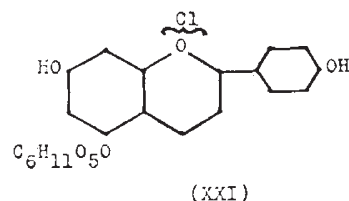

(XXI)

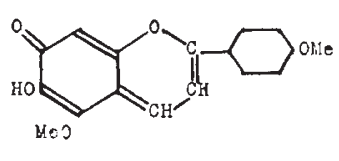

(XXII)
Among the matters of more general interest connected with the anthocyanins are the causes of colour variations in flowers, considered both statically and dynamically, that is, the actual condition of the pigment on one hand and its relation to genetic factors on the other. On the first aspect it may be noted that the pigments are indicators, the colours ranging from red oxonium salts to blue or violet salts of the colour-bases which are also acids. Thus cyanin chloride has a beautiful bluish-red colour, cyanin-base is violet and the potassium salt of cyanin-base is blue. Naturally, therefore, the colour of a flower is dependent on the $p \mathbf{H}$ of the cell-sap. But the range of $p \mathrm{H}$ is much smaller than experiments in vitro would suggest, and this is due to the combination of the anthocyanin colour-base with colloids tending to stabilise the anthocyanin anion at a $p \mathrm{H}$ which it could not survive in 'clean' solutions.

Another factor modifying the conclusions drawn from the indicator ranges observed in the labora- tory is the presence of co-pigments in the flowers. These are organic substances, mainly flavonols and tannins, which have a blueing effect on the colour irrespective of the $p \mathrm{H}$. The magnitude of the effect is, however, dependent on the $p \mathrm{H}$ and at a certain value becomes maximum. The phenomenon is the result of actual combination with the pigment and is accompanied by a marked change (diminution) of the distribution number.

A very characteristic reaction of cyanidin, petunidin and delphinidin derivatives is the deep blue ferric reaction. The presence of iron in the cell-sap might, therefore, be responsible for blue colours. Recent analysis of blue and red hydrangea flowers by Manly have been interpreted in this sense, and although this popular problem cannot be said to have been completely solved, the presence of iron and other metals must be reckoned with in considering the factors responsible for flower colours.

On the genetical aspect little need be said here; the subject deserves a separate treatment. Obviously the anthocyanin approach to the study of heredity represents one of the most promising lines of investigation, and it will be greatly facilitated by the chemical advances here briefly summarised. Miss Scott-Moncrieff and her colleagues have recently completed an investigation of the dahlias (private communication) which goes far to show that the pelargonin, eyanin and flavone or flavonols occurring in these flowers are biogenetically complementary and are phytosynthesised from a limited supply of protoflavan material. The full details will be studied with interest, and this type of investigation is pregnant with possibilities in connexion with the elucidation of the mechanism of anthocyanin synthesis in the plant.

I consider that the identification of the anthocyanin-chromogen with the flavones was an unfortunate obsession of the plant physiologists, and that in a different form the oxidase hypothesis of Keeble and Armstrong will be revived.

The leuco-anthocyanins of Laborde, Rosenheim and others will probably be found to be much nearer the mark. These substances are even more widely distributed than the anthocyanins (G. M. R. and R. R.), and their constitution may be foreshadowed by an investigation of peltogynol recently carried out in these laboratories (G. M. R. and R. R.). This is a constituent of certain woods known as 'purpleheart', and it is essentially a dihydrodesoxycyanidin condensed with formaldehyde. It is thought that the leuco-anthocyanins as a class may be of similar nature, the sensitive partly reduced flavylium nucleus being protected in a semi-acetal sugar-like structure. 